\title{
Inflación y crecimiento económico
}

\author{
Juan Carlos Moreno-Brid \\ Juan Carlos Rivas \\ Francisco G. Villarreal*
}

\begin{abstract}
Resumen
El artículo examina, con base en regresiones de panel, la relación de largo plazo entre la inflación y el crecimiento económico a partir de información para 70 países y datos anuales para 1950-2010. Los resultados no encuentran evidencia de un trade-off significativo entre inflación y crecimiento de largo plazo para dicha muestra y periodo, salvo si se incluyen experiencias nacionales con inflaciones anuales promedio por encima del umbral de $65 \%$ anual. Es decir, fuera de regímenes con tasas de inflación persistentemente muy elevadas, no hay una relación inversa sistemática ni significativa entre la inflación y el ritmo de crecimiento de la actividad productiva. La ausencia de dicho trade-off de largo plazo para el panel agregado dista de implicar que para cualquier economía individual la inflación mina su crecimiento sólo si ésta rebasa el 65\% anual. El análisis por grupos de países revela que el valor del umbral de significancia es función del régimen monetario en vigencia.
\end{abstract}

Palabras clave: trade-off, inflación y crecimiento económico, regresiones de panel, regímenes monetarios, economías de América Latina.

Clasificación JEL: C23, E31, O42.

\section{INTRODUCCIÓN}

La práctica convencional de la política macroeconómica desde la segunda mitad del siglo xx ha sido moldeada en buena medida por la convicción de que la inflación es un fenómeno monetario y que existe una relación inversa entre la inflación y el crecimiento de la economía en diferentes horizontes temporales.

Manuscrito recibido en marzo de 2014; aceptado en diciembre de 2014.

* Los autores están adscritos respectivamente a la sede subregional de la Comisión Económica para América Latina y el Caribe (CEPAL) en México, <JuanCarlos.MORENO@cepal.org>; BBVA Bancomer, México, $<$ jeancarlo_riv@hotmail.com>,y sede subregional de la cEPAL en México, < francisco.villarreal@cepal. org>. Las opiniones aquí expresadas son de su entera responsabilidad y no necesariamente coinciden con las de la Organización de las Naciones Unidas y de BBva Bancomer. Agradecemos los valiosos comentarios de los dictaminadores anónimos de la revista. 
$\mathrm{Al}$ respecto de esta relación, en la literatura se pueden identificar tres diferentes posturas.

La primera es que la inflación moderada puede tener un efecto favorable sobre la acumulación de capital debido al efecto de recomposición de cartera postulado por Tobin (1965). La idea básica de este enfoque, basada en el modelo de crecimiento de Solow (1956) y Swan (1956), es que los individuos mantienen dinero como un activo, por lo que incrementos en la inflación, al erosionar más rápido su valor, inducen una recomposición de cartera hacia la compra de activos físicos, maquinaria y equipo para actividades productivas. Dicha mayor acumulación de capital repercute en un aumento de la tasa de crecimiento económico en el corto plazo. ${ }^{1}$

Una conclusión en dirección similar se puede derivar de la noción de que el crecimiento requiere una transformación de la estructura productiva. Dicha transformación se acompaña de cambios favorables en los precios o rentabilidades relativas de diferentes industrias para atraer recursos — trabajo y capital-a actividades seleccionadas. Estas modificaciones en los precios relativos pueden empujar a un alza en el nivel de precios.

Una segunda postura sostiene que la inflación no tiene necesariamente impacto, ni favorable ni desfavorable, sobre el crecimiento económico en el largo plazo. Entre los proponentes, ya de buen tiempo atrás de esta postura, se encuentra Sidrauski (1967) que con base en un modelo de agentes optimizadores basado en el modelo de Ramsey (1928), donde la tenencia de dinero se motiva a partir del flujo de servicios provistos, sostiene que la tasa de acumulación de capital, y por lo tanto el crecimiento de la economía, es independiente de la tasa de inflación.

La tercera postura es que la inflación ineludiblemente es nociva para el crecimiento económico. ${ }^{2}$ Esta es la postura hoy en día más común, no sólo en la prensa de negocios sino entre un conjunto considerable de funcionarios a cargo

1 Algan y Ragot (2010) ilustran el efecto Tobin en un modelo teórico donde las decisiones de ahorro precautorio por parte de agentes que enfrentan incertidumbre en la ausencia de mercados completos, dan lugar a una relación positiva entre crecimiento e inflación, para niveles moderados de inflación. Por su parte, Mallik y Chowdhury (2001) encuentran evidencia empírica de una relación positiva entre ambas variables para el caso de cuatro países del sur de Asia.

2 Véase, por ejemplo, el trabajo de Andrés y Hernando (1999), quienes concluyen que existe una relación causal, de signo negativo y estadísticamente significativa, de la inflación hacia el crecimiento, que es robusta a la exclusión de episodios de alta inflación. 
de la conducción de la política macroeconómica en múltiples países. De hecho, esta posición tiende a ser la guía central de la conducta de los bancos centrales en nuestra región y con frecuencia se acompaña del supuesto explícito o implícito de que el crecimiento económico elevado y sostenido requiere como condición necesaria - y para algunos casos parecen argumentar que también suficiente- una inflación baja y estable.

En versiones menos estrictas de esta última postura se afirma que solamente cuando rebasa cierto umbral es que la inflación afecta significativa y adversamente la asignación eficiente de recursos, al punto de frenar la inversión y el ritmo de expansión económica (Sarel, 1995). De ser así, queda pendiente por identificar el umbral, en las comparaciones internacionales, a partir del cual la mayor inflación reduce el crecimiento de la economía. ${ }^{3} \mathrm{O}$ bien, ¿es que toda inflación, por baja que sea, es adversa al crecimiento? Esta es la pregunta que el presente artículo busca responder. Para ello se recurre al análisis estadístico comparativo de la relación entre las tasas medias de crecimiento del producto interno bruto (PIB) y del índice de precios al consumidor de las seis décadas recientes.

Así, para un número lo más elevado posible de economías, se analiza la significancia estadística de posibles umbrales a partir de los cuales sea válido concluir que, para la muestra de panel de los países considerados, hay una relación inversa entre la tasa de inflación y el ritmo de crecimiento económico. Cabe subrayar que dicho umbral se refiere a la muestra entera de países aquí incluidos y al periodo en cuestión, por lo tanto no representa el umbral individual que pueda o no tener cada economía en un momento dado o en la actualidad, a partir del cual si la inflación lo rebasa entonces el crecimiento de la actividad productiva correspondiente necesariamente perderá impulso.

En este empeño se parte de reconocer que los canales de transmisión, de interacción —o causalidad en ambos sentidos — entre la inflación y el crecimiento de la actividad productiva son complejos y variantes (Barro, 2013; Erosa y Ventura, 2002). Consideramos que son contingentes a diversas y cambiantes condicionantes estructurales, históricas e incluso a consideraciones de economía política expresadas, inter alia, en formas institucionales que inciden en los

3 En la literatura existen estimaciones bastante dispares de la magnitud de dicho umbral. Utilizando un panel de 87 países para el periodo 1970-1990, Sarel (1995) ubica el umbral en torno a una inflación anual de 5\%. En tanto que Bruno e Easterly (1998) ubican el umbral en torno a una inflación anual de 40\%, analizando episodios de alta inflación en un panel de 30 países a lo largo del periodo 1960-1994. 
mecanismos de fijación de precios y negociaciones salariales que no pueden ser articuladas o reflejadas de manera integral en un modelo analítico simple como el que aquí se introduce. De igual forma, en la interacción entre ambas variables puede influir marcadamente la conducción de la política económica, en particular en los casos en que la autoridad monetaria $-\mathrm{o}$ fiscal— vigila estrechamente la evolución de la inflación y actúa pronto y con firmeza con medidas de restricción crediticia, monetaria -0 de recorte al gasto- para contrarrestar alzas en la inflación.

En síntesis, el presente artículo hace un análisis sistemático tomando en cuenta información anual de 70 países para periodos de largo plazo de los coeficientes de correlación entre la inflación, medida como la mediana del índice de precios al consumidor de toda la muestra internacional, y del crecimiento económico, medido como la mediana de la tasa de variación del producto interno bruto real en dólares constantes. Este análisis toma en consideración diferentes rangos de inflación y distintas agrupaciones de países dependiendo de la naturaleza de su régimen monetario. Dicho análisis no encuentra evidencia — en la muestra conjunta de países y para el periodo seleccionado- de una correlación negativa (significativa generalizada) entre la inflación y el crecimiento económico, salvo cuando en la muestra se incluyen experiencias nacionales con inflaciones promedio de más de 65\% anual. Además, la evidencia aquí sistematizada indica que el valor preciso de dicho umbral de significancia es función del régimen monetario en vigencia.

El documento está organizado de la siguiente manera. Después de la presente introducción, la sección dos identifica la clasificación de regímenes monetarios que sirven de base para la agrupación de las economías en diferentes bloques para fines del análisis estadístico. En la sección tres se explora empíricamente, con métodos de panel, la relación entre inflación y crecimiento para la muestra de países seleccionados y agrupados en la forma descrita. La sección cuatro ofrece las conclusiones.

\section{REgíMENES DE POLÍTICA MONETARIA, INFLACIÓN Y CRECIMIENTO ECONÓMICO}

Para comenzar a contrastar la hipótesis de la existencia urbi et orbi de efectos significativos adversos de la inflación sobre el crecimiento económico es útil examinar los datos del diagrama de dispersión de la gráfica 1. Ésta reporta los 
valores de las medianas ${ }^{4}$ de la tasa anual de inflación y del crecimiento anual del PIB real de un panel de 70 países, con base en datos anuales del periodo 1951-2010. A simple vista, los datos del panel no indican una pauta clara de correlación -inversa o no- entre ambas variables. En cambio, presentan una gran concentración de observaciones en tasas relativamente bajas de la inflación anual con proporción alta de la muestra con su mediana correspondiente ubicada entre 1 y 10 por ciento.

\section{GRÁFICA 1}

Inflación y crecimiento económico en el mundo, 1951-2010

(mediana de la tasa media anual de crecimiento del pis real y de la inflación promedio anual, \%)

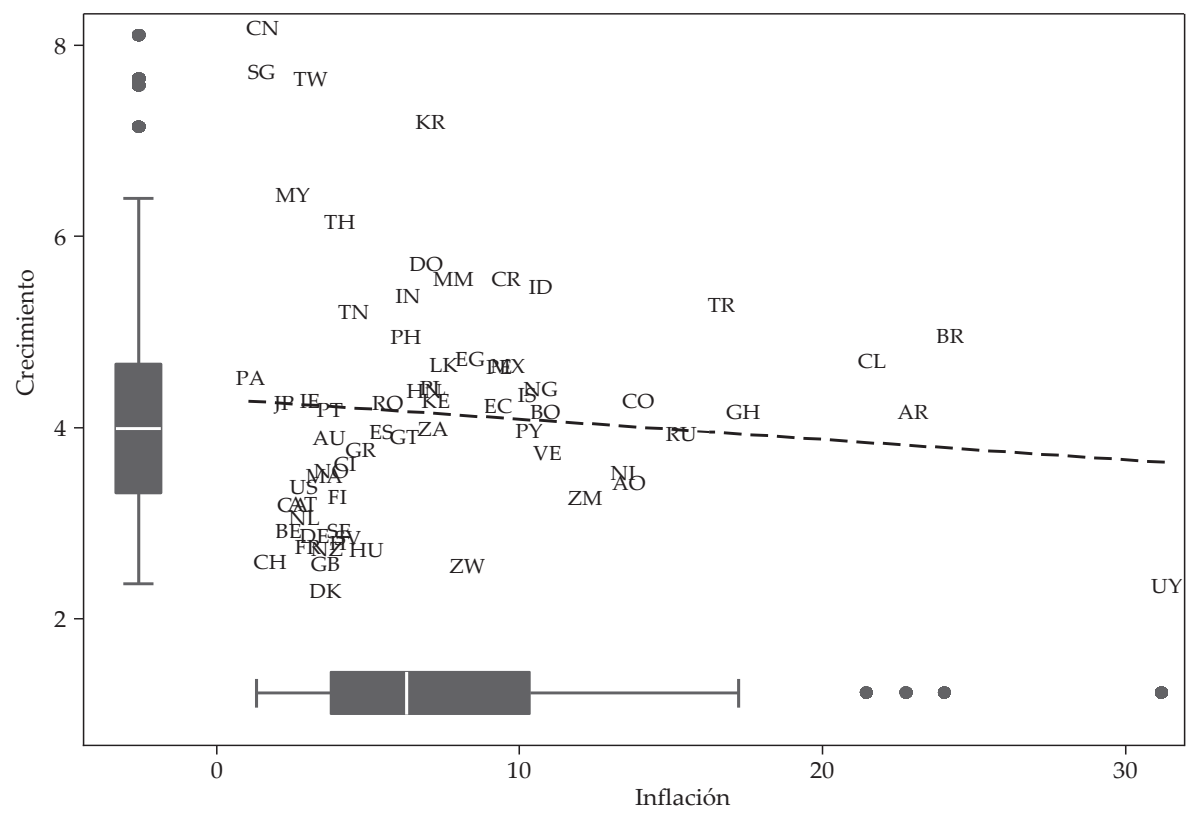

Notas: la ecuación de regresión es: crecimiento del PIB $=4.33-0.0210 *$ (inflación), donde el coeficiente de inflación tiene un error estándar de 0.0030 , el cual implica un estadístico $t$ de -7.3 . El coeficiente de determinación $\left(R^{2}\right)$ de la regresión lineal es de 0.0111 y y la raíz del error cuadrático medio es 1.2365. La muestra incluye 70 países, con base en datos del PIB real en dólares constantes e índices de precios al consumidor.

Fuente: elaboración propia con base en datos de Ilzetzki, Reinhart y Rogoff (2008), Reinhart y Rogoff (2010) y The Conference Board (2011).

4 Para cada país se calculan las medianas del crecimiento del PIB y la inflación de precios al consumidor a lo largo del periodo 1950-2011. Se prefiere la mediana como medida de tendencia central, ya que esta es menos sensible que la media a la ocurrencia de observaciones extremas. 
Para ahondar en ello, en la gráfica se incluye, asimismo, el resultado de la regresión lineal simple entre ambas variables estimada por mínimos cuadrados ordinarios. Esta línea, en contraste, sugiere una ligera correlación inversa entre las medianas de la inflación y del crecimiento, así como un incremento de 1\% en la mediana de la inflación anual asociado a una reducción de $2.1 \%$ en la mediana del crecimiento. No obstante que este resultado es estadísticamente significativo, con un valor del estadístico $t$ igual a 7.03, el ajuste de la regresión es muy pobre, ya que el coeficiente de determinación $\left(\mathrm{R}^{2}\right)$ es de apenas $1.13 \%$, y no pasa las pruebas convencionales de especificación correcta. De hecho, tanto en los ejes de la gráfica 1 y como en su diagrama de dispersión se incluyen diagramas de caja que dan cuenta formal de la distribución de ambas variables. ${ }^{5}$ Éstos apuntan que la distribución conjunta, y la correlación, entre ambas variables está influenciada por la ocurrencia de valores extremos.

En consecuencia, y para explorar de manera más sistemática la hipótesis sobre la significancia y dirección de la relación entre inflación y crecimiento económico, se procedió a segmentar la muestra con subgrupos de economías, en algún sentido homogéneas en cuanto a la conducción de su política monetaria. $\mathrm{Al}$ respecto, y si bien, tanto los objetivos como los instrumentos de la política monetaria han evolucionado, cabe aseverar que a lo largo del periodo analizado los bancos centrales han orientado dicha política a mantener la inflación al nivel más bajo posible. En este esfuerzo, en general su acción buscó proveer un ancla nominal para conformar las expectativas inflacionarias. En la adopción de dicha estrategia figura prominentemente la elección del régimen de tipo de cambio (véase, entre otros, White, 2013).

La gráfica 2 presenta la evolución de las medianas (de la muestra seleccionada de países) del crecimiento anual del PIB real y de la inflación a lo largo de 1951-2010. En primer lugar se evidencia un cambio importante en la tendencia de ambas variables a principios de la década de 1970, ligado al colapso del régimen de Bretton Woods y a los primeros choques petroleros. En efecto, el impacto de estos fenómenos se refleja en un alza en el nivel de inflación, así como en una baja en el ritmo de actividad económica mundial; cambios que marcaron el fin de las llamadas décadas doradas del desempeño de la econo-

\footnotetext{
5 Los extremos de los "rectángulos sólidos" dibujados en el eje vertical y en el horizontal muestran los percentiles 25 y 75 de la variable correspondiente, mientras que la línea interior muestra la mediana de la distribución. Por su parte, la terminación de las líneas en cada extremo de dichas cajas muestran los valores adyacentes, calculados como $3 / 2$ veces el rango intercuartil.
} 
mía que van desde la primera mitad de los años de 1950 hasta 1973. La gráfica indica, asimismo, cierta modificación de la relación entre la inflación y el ritmo de actividad económica. ¿Hasta qué grado se alteró de manera significativa la intensidad o dirección de la relación entre la inflación y crecimiento económico a nivel mundial? Es parte de las interrogantes para las cuales el presente trabajo aporta insumos para su respuesta. ${ }^{6}$

\section{GrÁfICA 2}

Inflación y crecimiento económico a nivel mundial, 1951-2010

(mediana del crecimiento anual del pis y de la inflación promedio anual, \%; muestra seleccionada de países)

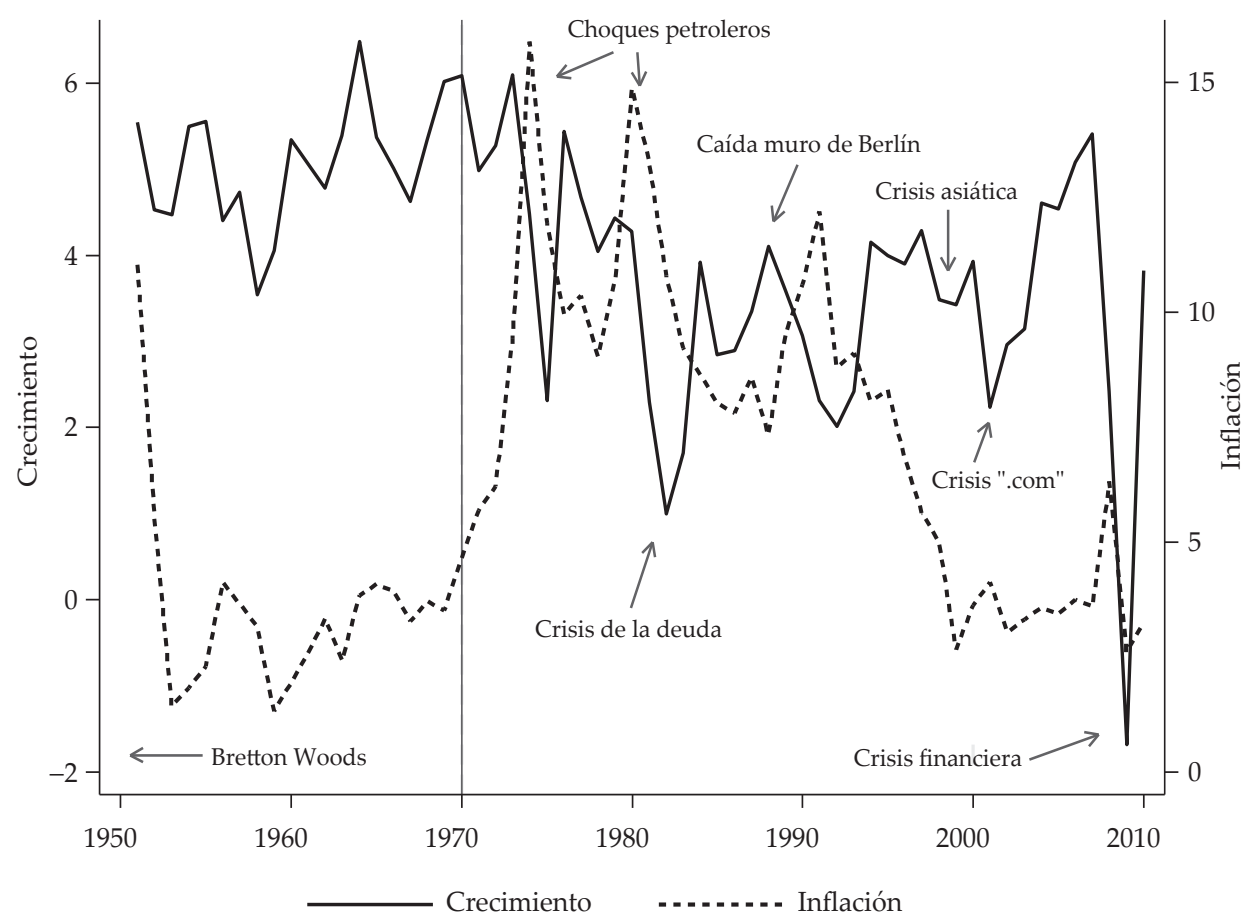

Fuente: elaboración propia con base en datos de Ilzetzki, Reinhart y Rogoff (2008), Reinhart y Rogoff (2010) y The Conference Board (2011).

${ }^{6}$ En la gráfica también se aprecia que, además de los choques de oferta producidos en los años de 1970 a raíz del embargo petrolero, las diversas crisis de los últimos 30 años han incidido en la evolución de la inflación y del crecimiento económico, y probablemente también en su interacción. 
La discusión anterior refleja en alguna medida las restricciones inherentes a lo que Fleming (1962) y Mundell (1963) llamaron la "Trinidad imposible", según la cual un país puede adoptar a lo más dos de las siguientes tres políticas: 1) libre movilidad de capitales en la balanza de pagos; 2) tipo de cambio nominal fijo, y 3) autonomía monetaria, entendida como la capacidad de fijar la tasa de interés nominal distinta a la que rige las transacciones en el mercado financiero internacional. De hecho, la evolución del régimen financiero internacional puede periodizarse según la ponderación asignada a cada una de estas tres políticas.

\section{RELACIÓN ENTRE INFLACIÓN Y CRECIMIENTO ECONÓMICO BAJO DIFERENTES REGÍMENES DE POLÍTICA MONETARIA: EVIDENCIA EMPÍRICA}

En la presente sección se explora la naturaleza de la relación entre inflación y crecimiento utilizando la taxonomía provista por las diferentes combinaciones de política provista en cuanto al régimen de tipo de cambio, el grado de apertura de la cuenta de capital y la autonomía monetaria.

En primer lugar se explora a profundidad la relación entre ambas variables a nivel agregado, tanto para la muestra global de países como la submuestra de países latinoamericanos, sin restricción alguna sobre el rango de las dos variables consideradas. Posteriormente, se repite el análisis de regresión pero restringiendo las muestras a fin de sucesivamente excluir de ella a las observaciones cuya inflación es extrema en el sentido de que rebasa ciertos rangos. Dichos rangos se van acotando a la baja de manera progresiva en cada ronda de estimación de los coeficientes de correlación a partir de los datos de panel.

Como se mencionó, las observaciones extremas en el panel de datos sesgan los coeficientes de correlación estimados. Una alternativa para corregir dicho sesgo es hacer el análisis estadístico con base en una transformación de las variables iniciales, que reduzca el efecto de las observaciones o valores extremos. Para el presente estudio se adoptó el procedimiento de sustituir la medición usual de la inflación anual del índice de precios al consumidor $(\pi)$ por su transformación no lineal que se identifica usualmente como una medición del impuesto inflacionario $[\pi /(1+\pi)]$.

El resultado del procedimiento anterior se presenta en la gráfica 3. El primer elemento a destacar es que la transformación propuesta atenúa el impacto de los valores extremos en la inflación. No obstante que el coeficiente de la regresión 
lineal sugiere la persistencia de una relación negativa entre las variables, que es estadísticamente significativa, el ajuste de la regresión es incluso peor que el observado antes de realizar la transformación. Para afinar el análisis, en el cuadro 1 se ofrecen los resultados de las regresiones de panel entre las observaciones de las medianas anuales de crecimiento del PIB y del impuesto inflacionario, restringiendo la muestra a diferentes niveles de inflación. El primer renglón reporta los resultados para el panel completo. El segundo reporta los resultados cuando la muestra se restringe a países de América Latina.

\section{GrÁFICA 3}

Impuesto inflacionario y crecimiento económico en el mundo, 1951-2010

(mediana de la tasa media anual de crecimiento del рів e impuesto inflacionario, \%)

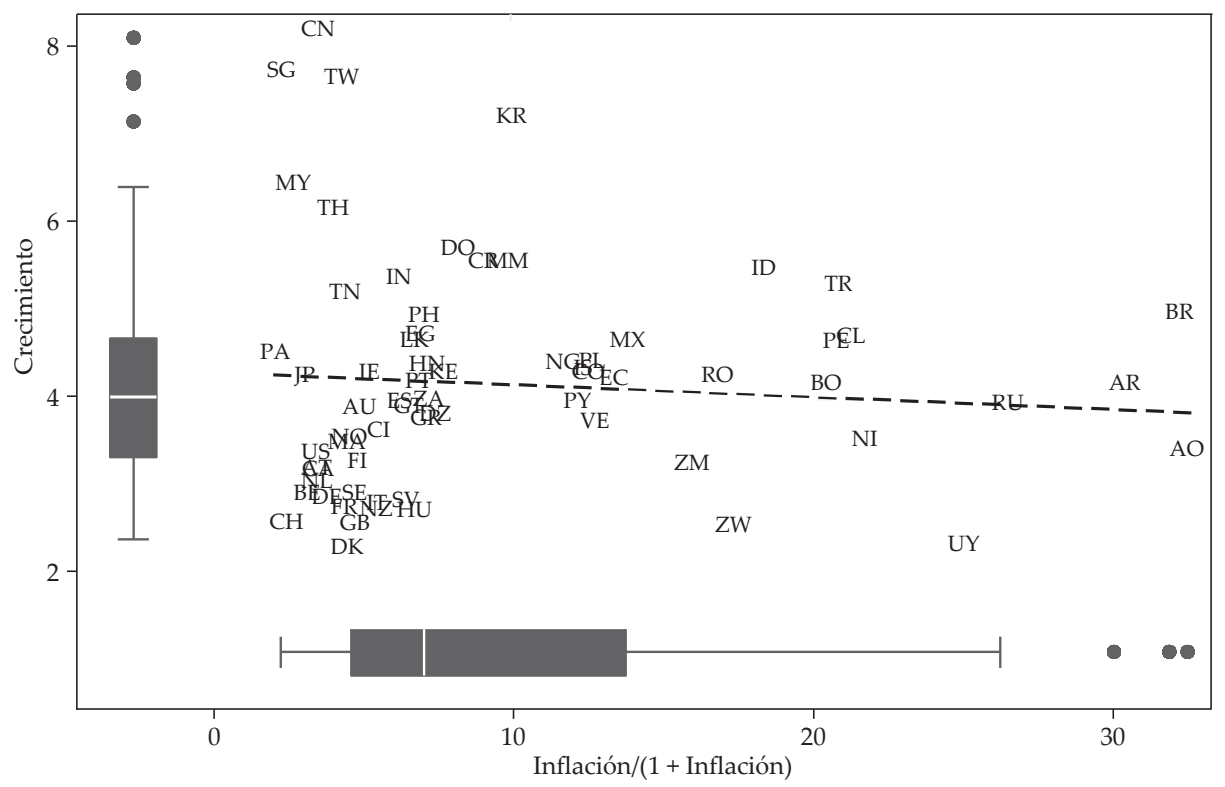

Notas: la ecuación de regresión es: crecimiento $=4.30-0.0138^{*}$ (impuesto inflacionario), donde el coeficiente de inflación tiene un error estándar de 0.0025, el cual implica un estadístico $t$ de -5.58 . El coeficiente determinación $\mathrm{R}^{2}$ de la regresión lineal es de 0.0071 , y la raíz del error cuadrático medio es 1.2391. La muestra incluye 70 países, con base en datos del PIB real en dólares constantes e índices de precios al consumidor.

Fuente: elaboración propia con base en datos de Ilzetzki, Reinhart y Rogoff (2008), Reinhart y Rogoff (2010) y The Conference Board (2011).

Es importante destacar el contraste con las regresiones de las gráficas 1 y 3. En éstas la mediana de cada país a lo largo del periodo representa una observación, 
y los coeficientes son estimados utilizando mínimos cuadrados ordinarios. En contraste, en el cuadro 1 se explota la información anual y la estructura de panel, por lo que para cada país y para cada año se tiene una observación, y los coeficientes se estiman utilizando regresiones de panel. ${ }^{7}$

\section{Cuadro 1}

Correlación entre impuesto inflacionario y crecimiento económico, 1951-2010

\begin{tabular}{llccccc}
\hline & \multicolumn{5}{c}{ Rango impuesto inflacionario } \\
\cline { 3 - 7 } & & Muestra & {$[-5,100]$} & {$[-5,80]$} & {$[-5,60]$} & {$[-5,40]$} \\
\hline 70 países & Coeficiente & $-0.0786^{* * *}$ & $-0.0802^{* * *}$ & $-0.0782^{* * *}$ & $-0.0715^{* * *}$ & $-0.0578^{* * *}$ \\
& Probabilidad & 0.00 & 0.00 & 0.00 & 0.00 & 0.00 \\
\multirow{2}{*}{ América Latina } & Coeficiente & $-0.733^{* * *}$ & $-0.0736^{* * *}$ & $-0.0716^{* * *}$ & $-0.0739^{* * *}$ & $-0.0577^{* * *}$ \\
& Probabilidad & 0.00 & 0.00 & 0.00 & 0.00 & 0.00 \\
\cline { 3 - 7 } & & {$[-5,20]$} & {$[-5,10]$} & {$[-5,5]$} & {$[-5,3]$} & \\
\cline { 3 - 7 } 70 países & Coeficiente & -0.0184 & 0.0435 & 0.1147 & 0.1091 & \\
& Probabilidad & 0.37 & 0.15 & 0.18 & 0.30 & \\
\cline { 3 - 7 } América Latina & Coeficiente & -0.0239 & -0.0067 & -0.1062 & 0.042 & \\
& Probabilidad & 0.52 & 0.94 & 0.54 & 0.87 & \\
\hline
\end{tabular}

Nota: los asteriscos en los coeficientes denotan su significancia estadística: ${ }^{* *} 99 \%,{ }^{* *} 95 \%,{ }^{*} 90 \%$. La cifra debajo del coeficiente indica la probabilidad con la que, bajo la hipótesis nula de que el coeficiente es igual a cero, se puede observar el valor del coeficiente estimado. Los rangos en paréntesis son sobre la inflación.

Fuente: elaboración propia con base en datos de Ilzetzki, Reinhart y Rogoff (2008), Reinhart y Rogoff (2010) y The Conference Board (2011).

Consistente con la evidencia mostrada en las gráficas 1 y 3 , con la transformación de la variable inflación, tanto para el panel de 70 países como para la submuestra de países de América Latina, los resultados de las regresiones confirman una relación inversa (es decir, un coeficiente negativo) entre el impuesto inflacionario y el crecimiento económico, estadísticamente significativa, para el conjunto completo del panel.

El cuadro 1, a partir de su segunda columna, ofrece el resultado del coeficiente de correlación y su significancia cuando se restringe la muestra a valores del impuesto inflacionario a rangos sucesivamente más estrechos. Esta sucesión se hace al eliminar las observaciones que rebasan los topes que van acotándose a la baja, comenzando con 100, hasta llegar a 3 en la última columna del cuadro,

7 Para todos los casos se estimaron los coeficientes utilizando tanto efectos fijos como efectos aleatorios; sin embargo, sólo se reporta el coeficiente seleccionado por el contraste de Hausman (1978). Los resultados de la selección del modelo no se reportan, pero están disponibles para consulta a través de los autores. 
en el bloque inferior. Se observa que en la medida en que los rangos del impuesto inflacionario se reducen para excluir las observaciones más elevadas, la magnitud del coeficiente estimado en la regresión se modifica, y también su nivel de significancia. Así, mientras que para la muestra completa el coeficiente estimado fue de -0.0786 , una vez que el tope máximo del rango se baja a 100 , el coeficiente estimado es de -0.0802 . Al continuar bajando el tope máximo, su magnitud en términos absolutos se reduce, hasta dar un valor estimado positivo cuando la cota máxima del rango de la muestra del impuesto inflacionario baja a 10 para el caso del panel completo de países, y a 3 para la muestra de países de América Latina.

Pero, más relevante que el cambio de signo del coeficiente estimado, es la modificación en su grado de significancia en ambos grupos a medida que los rangos del impuesto inflacionario van restringiéndose para excluir los valores extremos más elevados. En efecto, tanto en la muestra global compuesta por los 70 países como en la submuestra de países latinoamericanos, el coeficiente estimado deja de ser significativo una vez que el valor del impuesto inflacionario se restringe a un rango anual de entre -5 y 40 en ambas muestras. Es decir, cuando en la muestra se excluyen las experiencias con niveles medianos de la inflación superiores a $65 \%$, deja de percibirse en el panel — tanto total como el de países latinoamericanos- una correlación significativa inversa entre la inflación y la tasa de crecimiento económico.

Puesto de otra forma, la evidencia internacional para las últimas seis décadas, excluyendo las experiencias de economías que registraron inflaciones anuales superiores a $65 \%$, indica que no hay una relación estadísticamente significativa que revele que las economías con mayores niveles de inflación tendieran a registrar menores tasas de crecimiento del PIB real. El trade-off entre inflación y crecimiento simplemente no se observa de manera significativa en la comparación internacional a niveles de inflación menores a 65\% anual. Es decir, en este lapso economías con muy distintos niveles de inflación pueden haber registrado muy similares ritmos de expansión de su actividad productiva. No está demás subrayar que la ausencia de dicho trade-offen el panel de comparación internacional no implica necesariamente que en las economías individuales, en diferentes momentos, el alza aguda en su tasa de inflación no venga acompañada de una baja en su ritmo de crecimiento. Esto es un punto a investigar en el caso particular de cada economía en cuestión.

La sustitución aquí adoptada del indicador de la inflación por el del impuesto inflacionario reduce el sesgo provocado por las observaciones extremas, aún así 
la información contenida en 60 años del panel construido es muy heterogénea. Por su parte, el uso del panel completo para estimar las regresiones correspondiente no permite distinguir entre aquellos países que cuentan con una política monetaria autónoma y los que no. Se procedió a examinar la posibilidad de que la significancia, y quizás el signo, de la correlación entre ambas variables esté determinada por el tipo de política monetaria. Después de todo, es de esperar que en economías cuya política monetaria esté fuertemente orientada a abatir la inflación, las autoridades de la banca central respondan a episodios de alza en el índice de precios al consumidor con medidas contractivas del crédito que induzcan una pérdida de impulso en la actividad económica. La primera agrupación considerada en el presente estudio resultó de discriminar entre, por un lado, aquellos países que mantienen autonomía monetaria, ya sea adoptando un tipo de cambio flotante y una cuenta de capital abierta, o bien manteniendo controles en la cuenta de capital para preservar un tipo de cambio fijo; y, por otro, aquellos sin autonomía monetaria que, en general, mantuvieron un tipo de cambio fijo y una cuenta de capital abierta.

Dado que la adopción de tipos de cambio flotantes se generalizó a partir del colapso del régimen de Bretton Woods, en la gráfica 4 se presenta la evolución de la mediana de inflación y crecimiento a partir de 1970, clasificando las observaciones (país - año) en función de que cuenten o no con una política monetaria independiente. Lo primero que destaca es que los países sin autonomía monetaria tienen una inflación menor y menos volátil que los otros. Ello resulta paradójico en tanto que el objetivo principal de la mayoría de los bancos centrales es precisamente combatir la inflación. Además, y no obstante su menor nivel y menor variabilidad de la inflación, el crecimiento del pIB real en los países sin autonomía monetaria, con una mediana de $3.25 \%$, es tres cuartos de punto porcentual menor al de los países que cuentan con ella.

Ya que durante el periodo analizado la mayoría de países sin autonomía monetaria adoptaron tipos de cambio fijos respecto de las monedas de países desarrollados, el menor nivel de inflación refleja el beneficio de "importar" la inflación del país al cual está atado o referenciado su tipo de cambio nominal. Sin embargo, el menor crecimiento económico podría ser evidencia de la pérdida de competitividad de los países con tipo de cambio fijo ante la tendencia de apreciación real provocada por el llamado efecto Balassa-Samuelson (Balassa, 1964; Samuelson, 1964), en donde debido a incrementos en la productividad en el sector transable de los países en desarrollo se generan tasas de inflación mayores que las observadas en los países desarrollados. 


\section{GRÁFICA 4}

Evolución de la inflación y del crecimiento del PIB en grupos de países clasificados según su grado de autonomía monetaria, 1970-2007 (mediana del crecimiento y la inflación promedio anuales, \%)
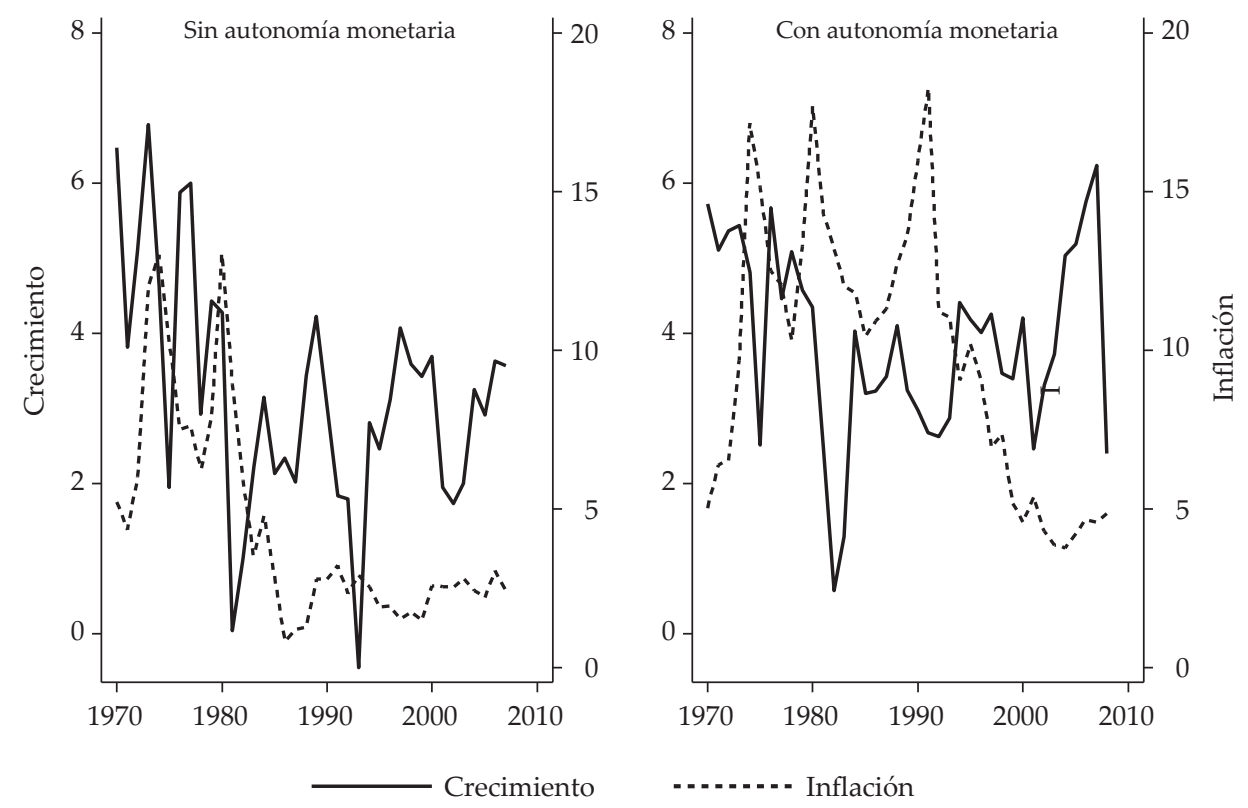

Fuente: elaboración propia con base en datos de Ilzetzki, Reinhart y Rogoff (2008), Reinhart y Rogoff (2010) y The Conference Board (2011).

En el bloque superior del cuadro 2 aparecen los coeficientes de correlación estimados entre la tasa de crecimiento y el impuesto inflacionario para el panel completo, discriminando según la independencia de la política monetaria; mientras que el bloque inferior ofrece los resultados utilizando solamente los datos de países de América Latina. Como en el caso anterior, las columnas presentan los coeficientes de correlación y su significancia en diferentes rangos del impuesto inflacionario.

El primer elemento que destaca es que, como se había mencionado, la inflación en los países sin autonomía monetaria es bastante menor que la observada en el grupo con autonomía monetaria, por lo que no es posible estimar la correlación en los rangos más amplios. Además, con excepción del rango más pequeño de América Latina, los coeficientes no son significativamente distintos de cero. En contraste, entre los países con autonomía monetaria se confirma 
la relación descrita anteriormente, donde tanto la magnitud de los coeficientes como su significancia se reducen en la medida que se restringe el rango de inflación, apuntando hacia la existencia de un umbral de significancia en la relación entre ambas variables.

\section{Cuadro 2}

Correlación entre impuesto inflacionario y crecimiento económico por grupo de país en cuanto a que tengan o no autonomía monetaria, 1970-2010

\begin{tabular}{|c|c|c|c|c|c|c|}
\hline \multirow[b]{2}{*}{ Rango } & & \multicolumn{5}{|c|}{ Panel 70 países } \\
\hline & & Muestra & {$[-5,100]$} & {$[-5,80]$} & {$[-5,60]$} & {$[-5,40]$} \\
\hline \multirow[t]{2}{*}{ Sin autonomía } & Coeficiente & $\ldots$ & $\ldots$ & $\ldots$ & $\ldots$ & -0.0012 \\
\hline & Probabilidad & $\ldots$ & $\ldots$ & $\ldots$ & $\ldots$ & 0.9820 \\
\hline \multirow[t]{3}{*}{ Con autonomía } & Coeficiente & $-0.084^{* * *}$ & $-0.0873^{* * *}$ & $-0.0906^{* * *}$ & $-0.0913^{* * *}$ & $-0.0827^{* * *}$ \\
\hline & Probabilidad & 0.0000 & 0.0000 & 0.0000 & 0.0000 & 0.000 \\
\hline & & {$[-5,20]$} & {$[-5,10]$} & {$[-5,5]$} & {$[-5,3]$} & \\
\hline \multirow[t]{2}{*}{ Sin autonomía } & Coeficiente & 0.0136 & 0.1057 & 0.0331 & 0.2331 & \\
\hline & Probabilidad & 0.8360 & 0.1210 & 0.8020 & 0.2190 & \\
\hline \multirow[t]{4}{*}{ Con autonomía } & Coeficiente & $-0.0456^{*}$ & 0.066 & $0.202^{*}$ & 0.2123 & \\
\hline & Probabilidad & 0.0640 & 0.2370 & 0.0720 & 0.4210 & \\
\hline & & \multicolumn{5}{|c|}{ América Latina } \\
\hline & & Muestra & {$[-5,100]$} & {$[-5,80]$} & {$[-5,60]$} & {$[-5,40]$} \\
\hline \multirow[t]{2}{*}{ Sin autonomía } & Coeficiente & $\ldots$ & $\ldots$ & $\ldots$ & $\ldots$ & -0.0034 \\
\hline & Probabilidad & $\ldots$ & $\ldots$ & $\ldots$ & $\ldots$ & 0.9390 \\
\hline \multirow[t]{3}{*}{ Con autonomía } & Coeficiente & $-0.07^{* * *}$ & $-0.0701^{* * *}$ & $-0.0678^{* * *}$ & $-0.07^{* * *}$ & $-0.053^{* *}$ \\
\hline & Probabilidad & 0.0000 & 0.0000 & 0.0000 & 0.0000 & 0.0430 \\
\hline & & {$[-5,20]$} & {$[-5,10]$} & {$[-5,5]$} & {$[-5,3]$} & \\
\hline \multirow[t]{2}{*}{ Sin autonomía } & Coeficiente & 0.0466 & 0.1762 & 0.347 & $1.0609^{* * *}$ & \\
\hline & Probabilidad & 0.6240 & 0.1750 & 0.2050 & 0.0080 & \\
\hline \multirow[t]{2}{*}{ Con autonomía } & Coeficiente & -0.0231 & 0.1581 & 0.1593 & 0.5617 & \\
\hline & Probabilidad & 0.6460 & 0.2670 & 0.7050 & 0.4940 & \\
\hline
\end{tabular}

Nota: los asteriscos al costado de los coeficientes denotan la significancia estadística: *** 99\%, ** 95\%, ${ }^{*} 90 \%$. La cifra bajo el coeficiente indica la probabilidad con la que, bajo la hipótesis nula de que el coeficiente es cero, se observe el valor estimado obtenido del coeficiente. Los rangos en paréntesis son sobre la inflación.

Fuente: elaboración propia en base a datos de Ilzetzki, Reinhart y Rogoff (2008), Reinhart y Rogoff (2010) y The Conference Board (2011).

Comparando los resultados del panel completo con los de América Latina, se puede observar que la magnitud de los coeficientes de correlación entre inflación y crecimiento económico es mayor en el panel completo. A la vez, mientras que en el panel completo la relación sigue siendo significativa en el rango de 
entre -5 y 20, este no es el caso para América Latina. Este resultado llama la atención, ya que históricamente la inflación ha sido considerada un tema de primera importancia en esta región. Una posible explicación es la presencia de otros factores que puedan estar afectando la interacción entre la inflación y el crecimiento, pero cuyo efecto no es capturado en este análisis.

Sin perjuicio de las estrategias que implican la ausencia de autonomía monetaria, es de particular interés estudiar con detalle los arreglos institucionales vigentes entre los países que han decidido mantener una política monetaria independiente. En la inmensa mayoría de estos casos, el principal objetivo de la política monetaria independiente es la búsqueda de la estabilidad de precios. Si bien, como argumentan Reinhart y Reinhart (2003), la solución al llamado trilema de las finanzas internacionales no necesariamente implica la adopción de regímenes puros. Con meros fines de exposición, a continuación se clasifica la muestra del panel de países durante los últimos cuarenta años de acuerdo a tres regímenes que representan extremos en el ámbito de las políticas posibles bajo el trilema.

En el primer régimen se combina un tipo de cambio nominal fijo con una cuenta de capital cerrada. Esta situación fue prevalente durante el régimen que Bretton Woods, vigente desde el final de la Segunda Guerra Mundial hasta 1971. El segundo régimen es el caso polar, donde se adopta un tipo de cambio flotante y se permite la libre movilidad de capitales, situación que en buena medida ha caracterizado al sistema financiero internacional desde mediados de la década de 1990. Por último, se considera un tercer régimen, en el cual se mantiene un tipo de cambio flotante, pero se mantienen las restricciones a la libre entrada y salida de capitales. Esta situación, aunque poco común actualmente, caracterizó a diversos países de América del Sur hasta finales de la década de 1990.

Para hacer operativa esta taxonomía, se utiliza la clasificación de regímenes de facto de tipo de cambio del Fondo Monetario Internacional (FMI) reportada por Ilzetzki, Reinhart y Rogoff (2008) y el índice de apertura de la cuenta de capital desarrollado por Chinn e Ito (2008). En particular, se clasifican como tipo flotante tanto los regímenes puros como los de flotación administrada; mientras que el índice de apertura de Chinn e Ito (2008) se normaliza para que sus valores se encuentren entre 0 y 1 , los cuales representan una cuenta de capital totalmente cerrada y abierta respectivamente, y se clasifica como abierta para aquellos casos donde el índice es mayor a 0.5. 
La relación del crecimiento económico y la inflación, a partir de esta taxonomía, se ilustra en la gráfica 5 . El primer elemento a destacar es la heterogeneidad dentro del grupo de países con autoridad monetaria. En este grupo, la mediana del crecimiento ha sido relativamente constante entre 3.5 y 4 por ciento a lo largo del periodo, pero las trayectorias bajo los distintos regímenes han sido bastante distintas. Por otra parte, para los tres regímenes se observa un importante descenso en la mediana de la inflación, que comenzó a observarse en la década de 1980 en los países con régimen II, y se consolida en un nivel inferior a 10\% anual en los otros dos regímenes hacia finales de la década de 1990. Empero, la experiencia previa al descenso generalizado de inflación es muy distinta, lo cual explica por qué con una mediana de $16.5 \%$ anual, la inflación bajo el régimen III es casi cuatro veces superior al $4.4 \%$ registrado por los países del régimen II.

\section{GRÁFICA 5}

Evolución de la inflación y el crecimiento económico por grupos de países según su régimen de autonomía monetaria, 1970-2007

(mediana del crecimiento y la inflación promedio anuales, \%)
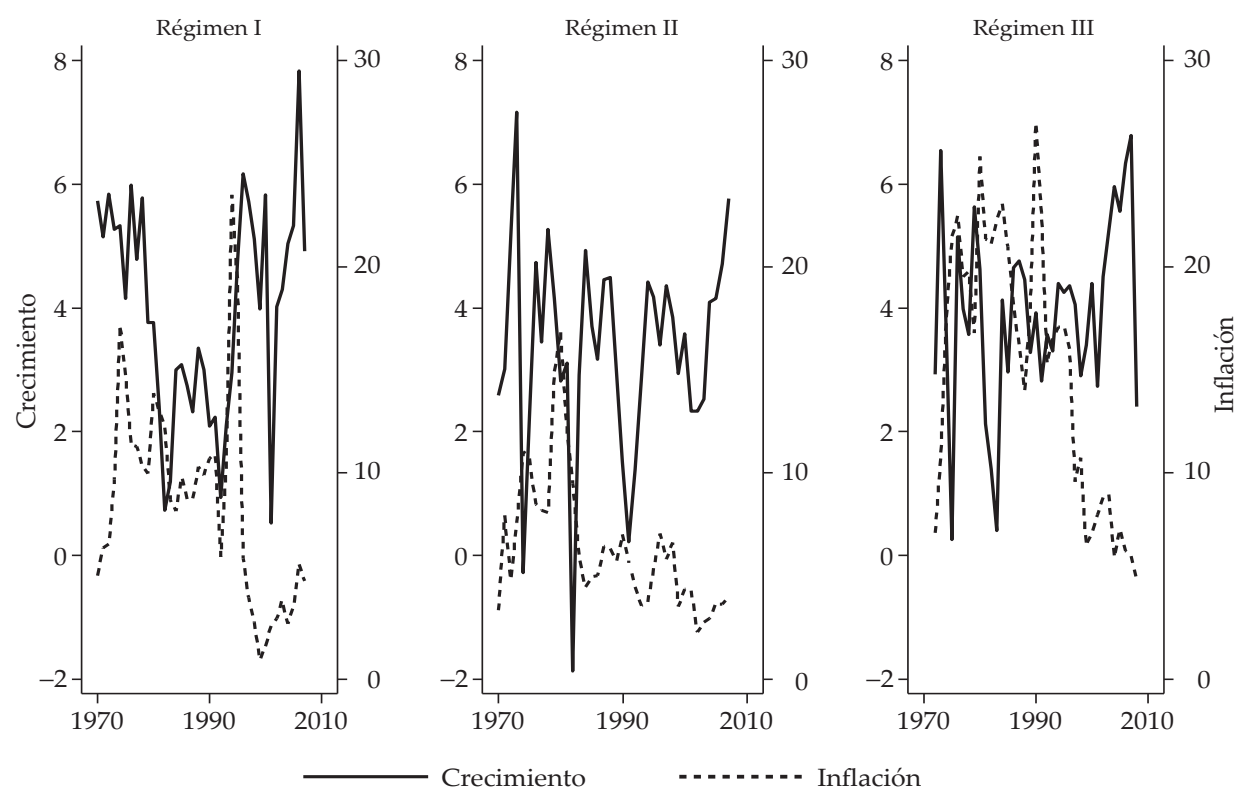

Nota: Régimen I. Tipo de cambio fijo y cuenta de capital cerrada; Régimen II. Tipo de cambio flexible y cuenta de capital abierta, y Régimen III. Tipo de cambio flexible y cuenta de capital cerrada.

Fuente: elaboración propia en base a datos de Ilzetzki, Reinhart y Rogoff (2008), Reinhart y Rogoff (2010) y The Conference Board (2011). 
Una posible explicación es que, como se mencionó, hasta la segunda mitad de la década de 1990 una parte significativa de los países bajo el régimen III son latinoamericanos, varios de los cuales sufrieron episodios de inflación muy alta. Entre ellos se destacan los casos de Argentina, Brasil y México durante la década de 1980.

El segundo rasgo que llama la atención es que entre los diferentes regímenes de autonomía monetaria no parece haber una correspondencia inversa entre la inflación y el crecimiento económico. En efecto, no obstante el relativamente bajo nivel de inflación que tuvieron, los países del régimen II registraron el menor ritmo de crecimiento económico entre los países con autonomía monetaria. Ello puede tener que ver con la volatilidad provocada por la libre movilidad de capital.

En el cuadro 3 se presentan los coeficientes de correlación de los tres grupos. Para el panel completo se observa que en los regímenes con cuenta de capital cerrada (I y III) se confirma una relación no lineal en la cual la magnitud y significancia de la correlación entre inflación y crecimiento se reduce en la medida que se excluyen los casos con niveles de inflación más elevada. A la vez, y como se destacó anteriormente, la evidencia indica que dicha relación es menos intensa para los países de América Latina. Es notable que bajo el régimen II, donde la autonomía monetaria se logra mediante la combinación de un tipo de cambio flexible y una cuenta de capital abierta, no parezca haber una relación inversa significativa entre inflación y crecimiento económico.

La literatura teórica de finales de 1960 argumentaba a favor de la autonomía monetaria en un entorno de tipos de cambio flexibles, con el argumento de que la política monetaria sería capaz de responder de manera adecuada ante choques heterogéneos. La conveniencia de este régimen estaba predicada en el mecanismo de estabilización provisto por la paridad descubierta de tasa de interés, según la cual las oportunidades de arbitraje provocadas por diferencias en las tasas de interés entre países serían eliminadas por ajustes en el tipo de cambio.

No obstante lo anterior, la volatilidad experimentada desde finales de la década de 1990, así como la evidencia empírica que indica que la paridad descubierta de la tasa de interés sólo se observa a lo largo de periodos muy largos, ha dado lugar a un replanteamiento de la conveniencia de adoptar posiciones extremas (soluciones de esquina) al trilema de las finanzas internacionales. De ahí se entiende aún más la atención de diversos países en la adopción de regímenes intermedios de tipo de cambio flotante, pero administrado, y medidas de gestión de los flujos de capital para garantizar una autonomía monetaria efectiva. 


\section{CuAdro 3}

Correlación entre impuesto inflacionario y crecimiento económico según régimen de autonomía monetaria, 1970-2010

\begin{tabular}{|c|c|c|c|c|c|c|}
\hline \multirow[b]{2}{*}{ Rango } & & \multicolumn{5}{|c|}{ Panel 70 países } \\
\hline & & Muestra & {$[-5,100]$} & {$[-5,80]$} & {$[-5,60]$} & {$[-5,40]$} \\
\hline \multirow[t]{2}{*}{ Régimen I } & Coeficiente & $-0.0889^{* * *}$ & $-0.0886^{* * *}$ & $-0.0893^{* * *}$ & $-0.0915^{* * *}$ & $-0.0892^{* * *}$ \\
\hline & Probabilidad & 0.0000 & 0.0000 & 0.0000 & 0.0000 & 0.0040 \\
\hline \multirow[t]{2}{*}{ Régimen II } & Coeficiente & $\ldots$ & $\ldots$ & $\ldots$ & $-0.0834^{*}$ & $-0.074^{* * *}$ \\
\hline & Probabilidad & $\ldots$ & $\ldots$ & $\ldots$ & 0.0860 & 0.0030 \\
\hline \multirow[t]{3}{*}{ Régimen III } & Coeficiente & $\ldots$ & $-0.0883^{* * *}$ & $-0.0973^{* * *}$ & $-0.1151^{* * *}$ & $-0.1332^{* * *}$ \\
\hline & Probabilidad & $\ldots$ & 0.0000 & 0.0000 & 0.0000 & 0.0000 \\
\hline & & {$[-5,20]$} & {$[-5,10]$} & {$[-5,5]$} & {$[-5,3]$} & \\
\hline \multirow[t]{2}{*}{ Régimen I } & Coeficiente & -0.0481 & 0.0544 & 0.1344 & 0.2899 & \\
\hline & Probabilidad & 0.3760 & 0.5180 & 0.6050 & 0.5050 & \\
\hline \multirow[t]{2}{*}{ Régimen II } & Coeficiente & -0.0597 & -0.0347 & -0.1477 & -0.0509 & \\
\hline & Probabilidad & 0.3270 & 0.6680 & 0.2550 & 0.8510 & \\
\hline \multirow[t]{4}{*}{ Régimen III } & Coeficiente & $-0.1444^{* * *}$ & $-0.2306^{*}$ & -0.1772 & -0.3246 & \\
\hline & Probabilidad & 0.0000 & 0.540 & 0.3160 & 0.2630 & \\
\hline & & \multicolumn{5}{|c|}{ América Latina } \\
\hline & & Muestra & {$[-5,100]$} & {$[-5,80]$} & {$[-5,60]$} & {$[-5,40]$} \\
\hline \multirow[t]{2}{*}{ Régimen I } & Coeficiente & $-0.0692^{* * *}$ & $-0.0699^{* * *}$ & $-0.0575^{* *}$ & -0.0472 & -0.0177 \\
\hline & Probabilidad & 0.0000 & 0.0000 & 0.0350 & 0.1290 & 0.6640 \\
\hline \multirow[t]{2}{*}{ Régimen II } & Coeficiente & $\ldots$ & $\ldots$ & $\ldots$ & -0.0706 & -0.016 \\
\hline & Probabilidad & $\ldots$ & $\ldots$ & $\ldots$ & 0.141 & 0.318 \\
\hline \multirow[t]{3}{*}{ Régimen III } & Coeficiente & $\ldots$ & $-0.0669^{* * *}$ & $-0.0711^{* * *}$ & $-0.0842^{* * *}$ & $-0.0974^{* *}$ \\
\hline & Probabilidad & $\ldots$ & 0.0020 & 0.0010 & 0.0000 & 0.0150 \\
\hline & & {$[-5,20]$} & {$[-5,10]$} & {$[-5,5]$} & {$[-5,3]$} & \\
\hline \multirow[t]{2}{*}{ Régimen I } & Coeficiente & -0.0022 & -0.1373 & -0.0973 & $\ldots$ & \\
\hline & Probabilidad & 0.9860 & 0.4850 & 0.9090 & $\ldots$ & \\
\hline \multirow[t]{2}{*}{ Régimen II } & Coeficiente & -0.0884 & -0.1321 & -0.3166 & $\ldots$ & \\
\hline & Probabilidad & 0.473 & 0.449 & 0.559 & $\ldots$ & \\
\hline \multirow[t]{2}{*}{ Régimen III } & Coeficiente & $-0.1148^{*}$ & -0.189 & 1.008 & $\ldots$ & \\
\hline & Probabilidad & 0.0580 & 0.7260 & 0.5460 & $\ldots$ & \\
\hline
\end{tabular}

Nota: los asteriscos al costado del coeficiente denotan su significancia estadística: ${ }^{* * *} 99 \%,{ }^{* *} 95 \%$, ${ }^{*} 90 \%$. La cifra debajo del coeficiente indica la probabilidad con la que, bajo la hipótesis nula de que el coeficiente es cero, se observe el valor estimado en la regresión. Los rangos en paréntesis son sobre la inflación anual.

Régimen I. Tipo de cambio fijo y cuenta de capital cerrada; Régimen II. Tipo de cambio flexible y cuenta de capital abierta, y Régimen III. Tipo de cambio flexible y cuenta de capital cerrada.

Fuente: elaboración propia en base a datos de Ilzetzki, Reinhart y Rogoff (2008), Reinhart y Rogoff (2010) y The Conference Board (2011). 


\section{Conclusiones}

Resumiendo, la evidencia examinada en el presente artículo, con base en regresiones de panel para una muestra de 70 países y datos anuales de 1950 a 2010, apoya la hipótesis de que existe una relación no lineal entre la inflación y el crecimiento económico, de manera que hay umbrales a partir de los cuales dicha relación inversa es estadísticamente significativa. Dicho umbral es relativamente alto, dada la experiencia reciente de la región, y parece depender del régimen monetario adoptado. Esta conclusión merece una serie de anotaciones adicionales importantes sobre sus implicaciones tanto en términos de la evolución económica de un país en particular como de carácter metodológico.

$\mathrm{Al}$ respecto, en primer lugar destaca que debido a que el presente análisis es un estudio de panel con una cobertura de 70 países, sus conclusiones no necesariamente se aplican a cada una de las economías individualmente. Para más precisión, el estudio concluye, con base en la muestra del panel mencionado de países y la información anual recabada para seis décadas, que no hay una relación inversa significativa entre la inflación de precios al consumidor y el crecimiento del PIB real, salvo cuando se incluyen experiencias con inflación anual superiores a 65 por ciento.

Dicho lo anterior, tal conclusión dista de querer implicar que para cualquier economía — dentro o fuera de la muestra — la inflación cobre relevancia, sea preocupante, para su crecimiento económico únicamente cuando rebase dicho nivel. Tal conclusión sería absurda. Nuestros resultados tan sólo indican que la experiencia internacional de las últimas seis décadas revela que, fuera de economías con tasas de inflación anual persistentemente por encima de $65 \%$, no hay una relación inversa sistemática ni significativa entre la inflación y el ritmo de crecimiento de la actividad productiva.

De hecho, nuestro análisis revela que la evidencia internacional de estos sesenta años registra múltiples y diversas experiencias nacionales con combinaciones de tasas de inflación y de ritmos de crecimiento económico, de manera que no necesariamente las economías que menos inflación registraron son las que más rápido se expandieron. El punto es especialmente relevante también para los casos en que aun estando en trayectorias bajas de inflación — digamos cercanas a $5 \%$ anual — se insiste en dedicar esfuerzos mayúsculos a disminuirla más bajo el supuesto de que ello es indispensable — necesario y suficiente- para detonar una senda de elevado y sostenido crecimiento. ¿Qué políticas macro y de trans- 
formación productiva son necesarias para asegurar la estabilización de precios, así como una expansión robusta y persistente de la actividad productiva? Es una pregunta relevante que a toda economía en América Latina le urge responder.

Una advertencia sobre el enfoque metodológico aquí adoptado es que al restringir la muestra, según regímenes de política y en rangos de inflación progresivamente más acotados, se excluye del análisis estadístico mucha información. Ello puede resultar en errores estándar demasiado grandes, o en coeficientes sesgados hacia cero. Una opción alternativa es la de utilizar regresiones cuantiles (Koenker y Bassett, 1978) y estimar la relación entre inflación y crecimiento para los percentiles de la distribución de inflación. Dicho enfoque permitiría tomar en cuenta toda la información, a la vez que controlar por diferentes características relevantes de las diferentes economías incluidas en el panel, como, por ejemplo, su régimen de política monetaria, la región geográfica a la que pertenecen o su nivel de ingreso, explotando la variación disponible en toda la muestra.

Asimismo, en nuestro esfuerzo por conmensurar la correlación entre dos variables cruciales en el comportamiento de toda economía - el ritmo de crecimiento y la inflación - se han dejado fuera del esquema muchos factores y consideraciones que seguramente inciden en los canales de interrelación entre ellas. Incorporarlos implicaría la construcción de un modelo macroeconométrico amplio, lo que rebasa con mucho los objetivos del presente estudio. Abordar estos retos metodológicos en el análisis de la inflación y el crecimiento será el objetivo de futuros trabajos.

\section{REFERENCIAS BIBLIOGRÁFICAS}

Algan, Y. y Ragot, X., 2010. Monetary Policy with Heterogeneous Agents and Borrowing Constraints. Review of Economic Dynamics, 13(2), pp. 295-316.

Andrés, J. y Hernando, I., 1999. Does Inflation Harm Economic Growth? Evidence for the OECD". En: Feldstein, M. (editor). The Costs and Benefits of Price Stability. Chicago IL: The University of Chicago Press, pp. 315-41.

Balassa, B., 1964. The Purchasing-Power Parity Doctrine: A Reappraisal. Journal of Political Economy, 72(6), 1 de diciembre, pp. 584-96.

Barro, R.J., 2013. Inflation and Economic Growth. Annals of Economics and Finance, 14(1), pp. 121-44.

Bruno, M. y Easterly, W., 1998. Inflation Crises and Long-run Growth. Journal of Monetary Economics, 41, pp. 3-26.

Chinn, M. e Ito, H., 2008. A New Measure of Financial Openness. Journal of Comparative Policy Analysis, 10(3), septiembre, pp. 309-22. 
Erosa, A. y Ventura, G., 2002. On Inflation as a Regressive Consumption Tax. Journal of Monetary Economics, 49(4), pp. 761-95.

Fleming, J.M., 1962. Domestic Financial Policies Under Fixed and Under Floating Exchange Rates. Staff Papers - International Monetary Fund, 9(3), noviembre, pp. 369-80. Hausman, J.A., 1978. Specification Tests in Econometrics. Econometrica, 46(6), pp. 1251-71. Ilzetzki, E., Reinhart, C.M. y Rogoff, K.S., 2008. Exchange Rate Arrangements Entering the 21 st. Century: Which Anchor Will Hold?" [mimeo]. Cambridge, MA: Harvard University.

Koenker, R. y Bassett, G., 1978. Regression Quantiles. Econometrica, 46(1), pp. 33-50. Mallik, G. y Chowdhury, A., 2001. Inflation and Economic Growth: Evidence from Four South Asian Countries. Asia Pacific Development Journal, 8(1), pp. 123-35.

Mundell, R.A., 1963. Capital Mobility and Stabilization Policy under Fixed and Flexible Exchange Rates. Canadian Journal of Economics and Political Science, 29, noviembre, pp.475-85.

Ramsey, F.P., 1928. A Mathematical Theory of Saving. The Economic Journal, 38(152), pp. 543-59.

Reinhart, C.M. y Reinhart, V.R., 2003. Twin Fallacies about Exchange Rate Policy in Emerging Markets [NBER Working Paper Series no. 9670]. National Burean of Economic Research (NBER), Cambridge, MA.

Reinhart, C.M. y Rogoff, K.S., 2010. From Financial Crash to Debt Crisis [NBer Working Paper Series no. 15795]. NBER, Cambridge, MA.

Sarel, M., 1995. Nonlinear Effects of Inflation on Economic Growth [IMF Working Paper no. WP/95/56]. International Monetary Fund (IMF), Washington DC.

Samuelson, P.A., 1964. Theoretical Notes on Trade Problems. The Review of Economics and Statistics, 46(2), 1 de mayo, pp. 145-54.

Sidrauski, M., 1967. Rational Choice and Patterns of Growth in a Monetary Economy. The American Economic Review, 57(2), 1 de mayo, pp. 534-44.

Solow, R.M., 1956. A Contribution to the Theory of Economic Growth. Quarterly Journal of Economics, 70(1), pp. 65-94.

Swan, T.W., 1956. Economic Growth and Capital Accumulation. Economic Record, 32(2), pp. 334-61.

The Conference Board, 2011. Total Economy Database. The Conference Board [en línea].

Disponible en: < https://www.conference-board.org/data/economydatabase/>. Tobin, J., 1965. Money and Economic Growth. Econometrica, 33(4), 1 de octubre, pp. 671-84.

White, W.R., 2013. Is Monetary Policy a Science? The Interaction of Theory and Practice Over the Last 50 Years [Globalization and Monetary Policy Institute Working Paper no. 155]. Federal Reserve Bank of Dallas, Dallas, Texas. 